\title{
Improving the diagnosis of cobalamin and related defects by genomic analysis, plus functional and structural assessment of novel variants
}

Sandra Brasil ${ }^{1 \dagger}$, Fátima Leal ${ }^{1 \dagger}$, Ana Vega ${ }^{1}$, Rosa Navarrete ${ }^{1}$, María Jesús Ecay ${ }^{1}$, Lourdes R. Desviat ${ }^{1}$, Casandra Riera², Natàlia Padilla², Xavier de la Cruz ${ }^{2,3}$, Mari Luz Couce ${ }^{4}$, Elena Martin-Hernández ${ }^{5}$, Ana Morais ${ }^{6}$, Consuelo Pedrón ${ }^{7}$, Luis Peña-Quintana ${ }^{8}$, Miriam Rigoldi ${ }^{9}$, Norma Specola ${ }^{10}$, Isabel Tavares de Almeida ${ }^{11}$, Inmaculada Vives ${ }^{12}$, Raquel Yahyaoui ${ }^{13}$, Pilar Rodríguez-Pombo ${ }^{1}$, Magdalena Ugarte ${ }^{1}$, Celia Pérez-Cerda', Begoña Merinero ${ }^{1 \dagger}$ and Belén Pérez ${ }^{1 * \dagger}$

\begin{abstract}
Background: Cellular cobalamin defects are a locus and allelic heterogeneous disorder. The gold standard for coming to genetic diagnoses of cobalamin defects has for some time been gene-by-gene Sanger sequencing of individual DNA fragments. Enzymatic and cellular methods are employed before such sequencing to help in the selection of the gene defects to be sought, but this is time-consuming and laborious. Furthermore some cases remain undiagnosed because no biochemical methods have been available to test for cobalamin absorption and transport defects.
\end{abstract}

Results: This paper reports the use of massive parallel sequencing of DNA (exome analysis) for the accurate and rapid genetic diagnosis of cobalamin-related defects in a cohort of affected patients. The method was first validated in an initial cohort with different cobalamin defects. Mendelian segregation, the frequency of mutations, and the comprehensive structural and functional analysis of gene variants, identified disease-causing mutations in 12 genes involved in the absorption and synthesis of active cofactors of vitamin $B_{12}$ (22 cases), and in the non-cobalamin metabolism-related genes ACSF3 (in four biochemically misdiagnosed patients) and SUCLA2 (in one patient with an unusual presentation). We have identified thirteen new variants all classified as pathogenic according to the ACGM recommendation but four were classified as variant likely pathogenic in MUT and SUCLA2. Functional and structural analysis provided evidences to classify them as pathogenic variants.

Conclusions: The present findings suggest that the technology used is sufficiently sensitive and specific, and the results it provides sufficiently reproducible, to recommend its use as a second-tier test after the biochemical detection of cobalamin disorder markers in the first days of life. However, for accurate diagnoses to be made, biochemical and functional tests that allow comprehensive clinical phenotyping are also needed.

Keywords: Cobalamin disorders, Methylmalonic aciduria, Homocystinuria, Massive parallel sequencing

\footnotetext{
*Correspondence: bperez@cbm.csic.es

†Sandra Brasil, Fátima Leal, Begoña Merinero and Belén Pérez contributed equally to this work.

${ }^{1}$ Centro de Diagnóstico de Enfermedades Moleculares, Centro de Biología

Molecular, Universidad Autónoma de Madrid, CIBERER, IdiPAZ, Madrid, Spain

Full list of author information is available at the end of the article
}

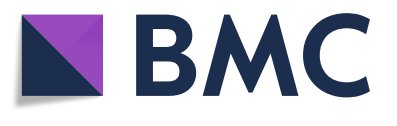

(c) The Author(s). 2018 Open Access This article is distributed under the terms of the Creative Commons Attribution 4.0 International License (http://creativecommons.org/licenses/by/4.0/), which permits unrestricted use, distribution, and reproduction in any medium, provided you give appropriate credit to the original author(s) and the source, provide a link to the Creative Commons license, and indicate if changes were made. The Creative Commons Public Domain Dedication waiver (http://creativecommons.org/publicdomain/zero/1.0/) applies to the data made available in this article, unless otherwise stated. 


\section{Background}

Cellular cobalamin problems are caused by nutritional deficiency or genetic defects that affect either the absorption or cellular uptake of the vitamin or the synthesis from it of methylcobalamin $(\mathrm{MeCbl})$ and adenosylcobalamin (AdoCbl). The latter are, respectively, a cofactor of methionine synthase (MS EC_2.1.1.13), which catalyzes the remethylation of homocysteine (Hcys) to methionine in the cytoplasm, and of methylmalonyl-CoA mutase (MUT EC_5.4.99.2), which catalyzes the mitochondrial isomerization of L-methylmalonyl-CoA (MMACoA) to succinylCoA. Defects in the synthesis of AdoCbl or the conversion of MMACoA to succinyl-CoA lead to elevated methylmalonic acid (MMA) concentrations, while defects in the synthesis of $\mathrm{MeCbl}$ or the remethylation of Hcys to methionine cause elevated Hcys (HC). Defects in the absorption and transport of vitamin $\mathrm{B}_{12}$, and in the cytosolic synthesis of the above cofactors, cause methylmalonic aciduria with homocystinuria (MMA\&HC) [1, 2].

After its intake, cobalamin is first bound to haptocorrin (encoded by TCN1) and then to the intrinsic factor (IF) encoded by GIF. To date, only one pathogenic mutation and one functional polymorphism (p.Asp301Tyr in TCN1) have been described [3, 4]. Cubilin and amnionless, encoded by $C U B N$ and $A M N$ respectively, form the cubam dimer which functions as the receptor of IF-Cbl in the ileum [4]. The IF is then degraded and vitamin $B_{12}$ appears in the blood stream associated with transcobalamin II (TCII) [2, 5]. Inherited malabsorption of cobalamin causes haematological and neurological abnormalities that can be fatal [6].

Still bound to TCII, the vitamin enters cells via transcobalamin receptor (TCblR)-mediated endocytosis. This receptor is encoded by $C D 320$, for which only one mutation has been described [7]. The cobalamin is then released into the cytosol, an event impaired in people with defects in LMBRD1 (cblF complementation group, MIM \#277380) [8]. LMBRD1 protein interacts with ABCD4 protein, which is also involved in the release of $\mathrm{Cbl}$ into the cytoplasm from the lysosomes (a defect which falls into complementation group cblJ) [9]. The most common form of cobalamin defect, complementation group cblC (MIM\#277400), is caused by mutations affecting MMACHC [10]. Patients with mutations in the Host Cell Factor 1 (HCFC1) locus (MIM 309541), located on the X chromosome, have a phenocopy of $c b l C$ disease [11] since $H C F C 1$ encodes a transcriptional co-regulator of $M M A C H C$. Recently additional defects have been described that affect transcription factors involved in the regulation of Cbl pathway: ZNF143 [12] and THAP11 [13]. In addition new variants in $P R D X 1$ also affect the expression of $M M A C H C$, named epi-cblC cases. Thus, these patients result from pathogenic variants in $M M A C H C$ and one in PRDX1 which force the antisense transcription of MMACHC and thereby a possible methylation mark [14].

Cellular cobalamin processing occurs via two major pathways - the cytosolic and mitochondrial pathways. The protein thought to be responsible for sorting cobalamin for these pathways is MMADHC (cblD complementation group, MIM \#611935). This complementation group is the most complex of all since patients present with biochemical heterogeneity ranging from isolated homocystinuria ( $c b l D$ variant 1) or isolated methylmalonic aciduria ( $c b l D$ variant 2) through to methylmalonic aciduria combined with homocystinuria ( $c b l D)$ depending on the nature and location of the mutations present [15].

The cytosolic pathway is involved in the synthesis of $\mathrm{MeCbl}$ by $\mathrm{MS}$, encoded by MTR (defects give rise to complementation group $c b l G$ ), while the enzyme methionine synthase reductase encoded by $M T R R$ is involved in the reactivation of MS (defects give rise to complementation group cblE). Both cblG (MIM\#250940) and $c b l E$ (MIM\#236270) defects, lead to elevated Hcys, hypomethioninaemia, and megaloblastic anaemia $[1,2]$.

Inactive cob(II)alamin entering the mitochondria is converted into AdoCbl via a reductive adenosylation reaction catalysed by an adenosyltransferase (ATR) encoded at the $c b l B$ locus (MIM\#251110) [16]. MUT is encoded at the MUT locus and two different phenotypes have been described: $m u t^{0}$ and $m u t^{-}$(MIM\#251100). Finally $M M A A$ is the gene associated with complementation group cblA defects (MIM\# 607481). It encodes a protein of the same name which may be involved in transferring AdoCbl from ATR to MUT protein as well as maintaining MUT's functional integrity $[5,17]$.

Mildly elevated MMA also occurs in patients with mutations affecting SUCLA2 (MIM\#612073) and SUCLG1 (MIM\#245400) which lead to infantile mitochondrial encephalopathic depletion syndrome. These genes encode either the $\beta$-subunit of ADP-forming or the $\alpha$-subunit of GDP-forming succinyl-CoA synthetases. The accumulated succinyl-CoA inhibits the metabolism of MMACoA to succinyl-CoA, leading to the accumulation of MMA in body fluids. Mutations in SUCLA2 give rise to typical (though rare) early onset dystonia combined with deafness $[18,19]$. An increase in MMA is also seen in patients with mutations in ACSF3 (MIM\# 614265), in whom malonic acid levels may also be elevated (combined malonic and methylmalonic aciduria, CMAMMA) [20]. ACSF3 encodes a mitochondrial acyl-CoA synthetase considered to have malonyl-CoA and MMACoA synthetase activities.

The diagnosis of an intracellular cobalamin metabolism disorder in a symptomatic individual is based on both clinical suspicion and biochemical analyses [1, 21]. Since expanded newborn screening potentially allows 
early detection of certain disorders of intracellular cobalamin metabolism, some affected individuals may be diagnosed prior to the onset of clinical symptoms. Diagnosis is based on elevated propionylcarnitine (C3), a high propionylcarnitine to acetylcarnitine ratio $(\mathrm{C} 3 / \mathrm{C} 2)$, high heptadecanoylcarnitine (C17), and/or reduced methionine concentrations [22]. After symptomatic or asymptomatic detection, a differential diagnosis is needed to help identify the gene that might be involved. Confirmatory testing is based on measuring Hcys and MMA in plasma and/or urine, enzyme analyses, the incorporation of $\left[1-{ }^{14} \mathrm{C}\right]$ propionate and $\left[5-{ }^{14} \mathrm{C}\right]$ methylTHF into proteins in fibroblasts cultured in basal and hydroxocobalamin-supplemented media, and in some cases by cellular complementation assays [1]. However, biochemical analysis cannot pinpoint a genetic defect, making diagnosis complicated. Fortunately, recent developments in high-throughput sequence capture have made next generation sequencing (NGS) a rapid and accurate means of analysing genetic locus and allele heterogeneous disorders [23-25].

The main purpose of clinical laboratory testing is to support medical decision-making. Clinical genetic testing is generally used to identify or confirm the cause of a disease and, if possible, to provide ideas for personalized treatment. Massive parallel sequencing is the starting point of a complex process for identifying pathogenic variants as well as the description of new functions for a gene product [25]. The aim of the present work was to assess the use of genetic studies as second-tier tests following the detection of cobalamin disorders in biochemical newborn screening. The usefulness of massive parallel sequencing in the identification of the mutated genes responsible for cobalamin and related defects was analyzed, and the structural and functional analysis of the novel variants identified undertaken.

\section{Methods \\ Patients}

Patient-derived fibroblasts, blood samples or dried blood spots from 27 patients with elevated MMA and/or Hcys or vitamin $B_{12}$ deficiency were referred to our laboratory for genetic analysis. Another 9 samples from patients with cobalamin defects, previously diagnosed in our laboratory, were included for validation purposes. The diagnosis of these cases was made by biochemical, cellular and genetic studies. Fourteen cases were detected through expanded newborn screening programs; the others were diagnosed after clinical presentation (neonatal or late onset). The study was approved by the ethics committee of the Universidad Autónoma de Madrid. The participants or their legal guardians gave their signed, informed consent to be included.

\section{Genetic analysis}

High purity DNA was extracted from whole blood, fibroblasts or dried blood spot samples using the MagNA Pure Compact Kit (Roche Applied Biosciences, Indianapolis, IN) following the manufacturer's protocol. Two massive parallel sequencing panels were used: targeted customized exome sequencing to capture the exome of 120 genes involved in metabolic disorders (Nextera Nature Capture, Illumina, San Diego, California, USA) (the list of genes included can be sent upon request), and an extended panel (Clinical-Exome Sequencing TruSight ${ }^{\mathrm{m}}$ One Gene Panel, Illumina) that included all the known (in 2013) disease-associated genes described in the OMIM database (Mendeliome panel). For genetic analysis in the validation cohort both panels were used. For genetic analysis in discovery cohort either of the two panels was used, except in cases P24-P27 where both panels were used. After gene capture and alignment with the reference genome, variant callings were made using a virtual panel that included all cobalamin metabolism and related genes. Incidental findings in genes unrelated to the clinical/biochemical phenotypes were ignored. Additional files 1 and 2 describes the coverage of the genes. With both panels, the libraries generated were sequenced using 150 bp paired-end reads employing the Illumina MiSeq or Nextseq500 NGS platforms. SNVs was annotated and subsequently filtered as previously described [26]. Variants were always confirmed by conventional Sanger sequencing employing both patient genomic DNA and that of their parents if available. Pathogenic prediction was assessed by Alamut Visual software. Variants were classified following the ACMG recommendation guidelines $[27,28]$.

\section{Assessment of variant pathogenicity}

To assess the isolated effect of the recently described mutation c.1084-10A > G in MUT [29], the splicing profile was analyzed ex vivo using a minigene assay. Briefly, exon 6 and the corresponding flanking intronic regions from patient P10 were amplified as previously described and cloned into pSPL3 vector [30]. Transcriptional profile analysis was performed as described elsewhere [30].

The oxygen consumption rate (OCR) was measured using the XF24 Extracellular Flux Analyzer (Seahorse Bioscience, Billerica, MA, USA) as previously described [31] in cells transduced with lentiviral particles incorporating wild-type cDNA of SUCLA2 (NM_003850) or the respective negative control. Modulating compounds such as oligomycin inhibitor of the ATP synthase (OL, $6 \mu \mathrm{M}$ ), carbonyl cyanide 4-(trifluoromethoxy) phenylhydrazone a potent uncoupler of oxidative phosphorylation in mitochondria (FCCP, $50 \mu \mathrm{M})$, rotenone inhibitor of complex I (RO, $1 \mu \mathrm{M})$ and antimycin A inhibitor of complex III (AT, $1 \mu \mathrm{M}$ ) were used to assess the 
bioenergetic profile and were sequentially added to the cells. The calibration plate for these compounds was prepared according to the manufacturer's protocol. The data obtained were used to determine basal OCR, the maximum respiratory rate, ATP-linked respiration, mitochondrial reserve capacity, proton leakage and non-mitochondrial respiration. Briefly, for OCR determinations, patient-derived skin fibroblasts $\left(5 \times 10^{4}\right.$ each), were seeded per well in 24-well tissue culture microplates and incubated with $100 \mu \mathrm{L}$ DMEM supplemented galactose $(1 \mathrm{~g} / \mathrm{L} \mathrm{g} / \mathrm{l}$, DMEM-Gal) instead of glucose at $37{ }^{\circ} \mathrm{C}$ for $2-5 \mathrm{~h}$. After the incubation period, up to $250 \mu \mathrm{l}$ of the corresponding medium was added and incubated overnight at $37{ }^{\circ} \mathrm{C}$. On the next day, the medium was removed and the cells washed with phosphate saline buffer (PBS). $700 \mu \mathrm{l}$ of the corresponding medium DMEM-Gal without bicarbonate were then added and the plates incubated at $37^{\circ} \mathrm{C}$ for $1 \mathrm{~h}$.

\section{Functional prediction}

The novel intronic and exonic SNVs identified were analyzed using Alamut Visual software, which includes a number of functional predictors. The two SUCLA2 variants (p.Gly326Arg, p.Ile312Thr) were mapped to a homology model of the human protein based on the known X-ray structure of SUCLG2 (S. scrofa; PDB code: 2fp4) [32]. The percentage sequence identity between these proteins was $54 \%$ [33].

\section{Results}

\section{Genetic analysis}

To assess the sensitivity of the proposed assay in the detection of pathogenic mutations, all mapped sequence reads from the nine samples (VC1-9) with previously defined mutations in TCN2, MTR, MTRR, MMACHC, $M M A D H C, M U T, M M A A$ and $M M A B$ (Table 1) (11 known and three unknown mutations detected by conventional Sanger sequencing) were inspected blind. Samples for the validation cohort were selected to include different types of $\mathrm{Cbl}$ deficiency. All the previously known mutations in all samples were detected in their correct heterozygous/homozygous state.

The discovery cohort consisted of samples received for cobalamin genetic diagnosis, for which no mutations were previously known. Variant changes were identified in the cobalamin transport genes i.e., TCN1, GIF and $A M N$, in genes causing isolated MMA i.e., $M M A A$, $M U T$ and $M M A B$, in genes causing MMA\&HC i.e., CD320, MMACHC, MMADHC, in genes causing $\mathrm{HC}$ i.e., $M T R R$, and in two MMA metabolism-related genes SUCLA2 and ACSF3 (Table 1). Some variants in $M M A C H C, M M A D H C, M U T, M T R R$ and ACSF3 were detected in several samples. In some cases DNA from blood spots was used in parallel; the coverage compared to whole blood was similar.

Overall, 33 variations were identified in 12 different genes. All the new exonic non-synonymous SNVs (nsSNVs) were predicted to be pathogenic or likely pathogenic (Additional files 1 and 3).

Among 27 samples with biallelic nucleotide changes, 21 showed at least one described $\left(\mathrm{HGMD}^{\circ}\right.$ professional release professional release 2018.1) or one new LoF mutation. Three samples (P6, P9 and P23) presented two new variants in $M U T$ or SUCLA2. A previously described functional SNP in TCN1 was detected in two samples (P1 and P2) [4]. Although this disease-associated polymorphism was not detected in the in-house control samples analysed, this variation is present close to $9 \%$ in all data base consulted (ExAc, gnomAD and Spanish data) with presence of homozygous cases. Therefore this change alone in TCN1 is unlikely to be responsible for the clinical picture in $\mathrm{P} 1$ and $\mathrm{P} 2$.

P11 (identified by newborn screening) presented the previously described nonsense mutation p.Arg152Ter in $M U T$, plus the disease-associated polymorphism in TCN1 described above. Both changes were present in the paternal sample. No other exonic or described intronic mutation were detected. Mean coverage allowed to rule out a large genomic deletion. It was not possible to rule out the presence of deep intronic mutations.

Four patients (P24, P25, P26 and P27) referred for analysis of genes related to isolated MMA in fact had mutations in ACSF3. After the genetic diagnosis, a slight increase in urinary malonic acid was confirmed by GC-MS in three of them.

\section{Functional analysis of the MUT splicing mutation c.1084- $10 \mathrm{~A}>\mathrm{G}$}

The effect of mutation c.1084-10A $>$ G identified in P10 on the splicing process was addressed using an ex vivo minigene system. The results indicate the insertion of a 9 nt sequence upstream of the normal 3' splice site, as recently described by transcriptional profile analysis in patient-derived fibroblasts [29]. A shorter transcript was also observed, resulting from the deletion of $135 \mathrm{nt}$ from exon 6 . This was detected as a heteroduplex along with the normal transcript. This deletion was generated by the activation of an internal exonic cryptic 3'splice with a higher splicing score than normal, plus two exonic splicing enhancer sequences (Fig. 1).This deletion would likely translate into the elimination of 45 amino acid residues from the protein.

\section{Functional analysis of SUCLA2 defects in patient-derived fibroblasts}

To assess the bioenergetic profile of patient P23, and to determine whether mitochondrial function could be 
Table 1 Genetic and biochemical findings of validation and discovery cases

\begin{tabular}{llll}
\hline Reference & Gene & $\begin{array}{l}\text { Allele 1 Nucleotide change } \\
\text { (protein effect) HGMD number }\end{array}$ & $\begin{array}{l}\text { Allele 2 Nucleotide change } \\
\text { (protein effect) HGMD number }\end{array}$ \\
\hline VC1 & TCN2 $^{a}$ & $\begin{array}{l}\text { c.497_498delTC (p.Leu166Profs*7) } \\
\text { CD106933 }\end{array}$ & $\begin{array}{l}\text { C.497_498delTC } \\
\text { (p.Leu166Profs*7) CD106933 }\end{array}$
\end{tabular}

Clinical and biochemical findings

c.497_498deITC (p.Leu166Profs*7) (p.Leu166Profs*7) CD106933

$2 \mathrm{~m}$ pancytopenia, $\mathrm{FTT}$, metabolic acidosis. Improvement after im $\mathrm{OHCbl}$

$\uparrow$ Hcys (pl): $26 \mu \mathrm{mol} / \mathrm{L} ; \uparrow C 3: 3.4 \mu \mathrm{mol} / \mathrm{L} ; \uparrow M M A$ (ur): $419 \mathrm{mmol} / \mathrm{mol}$ creatn

Propionate uptake (FB) $(\mathrm{nmol} / 10 \mathrm{~h} / \mathrm{mg}$ prot):

Normal

-OHCbl (patient/control): 0.76/0.97

+OHCbl (patient/control): 0.98/0.83

$\begin{array}{llll}\text { VC2 } & \text { MTR } & \text { c.1348_1349delTCinsGA (p.Ser450Asp) } & \text { c.3008-4A > G (p.?) CS135282 } \\ \text { VC3 } & \text { MTRR } & \text { c.1361C > T (p.Ser454Leu) CM032288 } & \begin{array}{l}\text { c.1769+ 1G > A } \\ \text { (p.Glu560_Arg590del) }\end{array} \\ \text { VC4 } & \text { MMACHC } & \text { c.271dupA (p.Arg91Lysfs*14) Cl055013 } & \begin{array}{l}\text { c.271dupA (p.Arg91Lysfs*14) } \\ \text { Cl055013 }\end{array} \\ \text { VC5 } & \text { MMACHC } & \text { c.271dupA (p.Arg91Lysfs*14) Cl055013 } & \begin{array}{l}\text { c.626dupT (p.Thr210Aspfs*35) } \\ \text { Clo95519 }\end{array}\end{array}$

$2 \mathrm{~m}$ apnea, somnolence, hypotonia, seizures $\uparrow$ Hcys (pl): $79 \mu \mathrm{mol} / \mathrm{L} ; \mathrm{MMA}$ (ur) (N)

MGA, low serum vit B12; $\uparrow$ Hcys (pl): $90 \mu \mathrm{mol} / \mathrm{L} ; \mathrm{N}$ MMA (ur)

NBS: $\uparrow C 3+C 3 / C 2$ in DBS

$\uparrow$ Hcys (pl); $\uparrow$ MMA (ur) > $1000 \mathrm{mmol} / \mathrm{mol}$ creatn

NBS: $\uparrow C 3$ in DBS

$\uparrow$ Hcys: $159 \mu \mathrm{mol} / \mathrm{L} ; \mathrm{C} 3$ (pl): $8.2 \mu \mathrm{mol} / \mathrm{L} ; \uparrow M M A$ (ur): $608 \mathrm{mmol} / \mathrm{mol}$ creatn

Propionate uptake (FB) (nmol/10 h/mg prot):

-OHCbl (patient/control): 0.23/1.90

$+\mathrm{OHCbl}$ (patient/control): 2.3/2.34

c.57_64del8 (p.Ser20*) CD082071

4d hypotonia, metabolic acidosis, hyperammonemia

N Hcys (pl); $\uparrow M M A$ (ur): $5875 \mathrm{mmol} / \mathrm{mol}$ creatn; Propionate uptake (FB) $(\mathrm{nmol} / 10 \mathrm{~h} / \mathrm{mg}$ prot): -OHCbl (patient/control): 0.13/3.31

+OHCbl (patient/control): 2.30/3.37 Mutase (FB):N

$\begin{array}{lll}\text { VC7 } & \text { MUT }^{a} & \text { c.312delC (p.Trp105Glyfs*75) } \\ \text { VC8 } & \text { MMAA }^{a} & \text { c.64C > T (p.Arg22Ter) CM042745 }\end{array}$

c.570 572dupCCG (p.Arg191dup) Cl154655

$\begin{array}{lll}\text { P1 } & \text { TCN1 } & \text { c.901G }>T^{\mathrm{b}} \text { (p.Asp301Tyr) CM099555 } \\ & & \\ \text { P2 } & \text { TCN1 } & \text { C.901G }>T^{\mathrm{b}} \text { (p.Asp301Tyr) CM099555 } \\ \text { P3 } & \text { GIF } & \text { c.389C > G (p.Ser130Ter) } \\ & & \\ \text { P4 } & \text { AMN }{ }^{a} & \text { C.514-34G > A (p.Thr172fs) CS127867 } \\ & & \\ \text { P5 } & \text { MMAA } & \begin{array}{l}\text { C.593_596delCTGA (p.Thr198Serfs*6) } \\ \text { CD043681 }\end{array} \\ \text { P6 } & \text { MUT } & \text { c.904G > C (p.Ala302Pro) }\end{array}$

c.901G > T $T^{\mathrm{b}}$ (p.Asp301Tyr) CM099555

c.901G > T (p.Asp301Tyr) CM099555

c.389C > G (p.Ser130Ter)

c.1046C > A (p.Ser349Ter)

c.593_596delCTGA (p.Thr198Serfs*6) CD043681

c.904G > C (p.Ala302Pro)
$12 \mathrm{~m}$ metabolic acidosis $\uparrow M M A$ (ur): $8810 \mathrm{mmol} / \mathrm{mol}$ creatn

$5 \mathrm{~m}$ metabolic acidosis. Improvement after im $\mathrm{OHCbl}$

$\uparrow M M A$ (ur)

Propionate uptake (FB) (nmol/10 h/mg prot): -OHCbl (patient/control): 0.12/1.32 $+\mathrm{OHCbl}$ (patient/control): 0.42/2.17 Mutase (FB): N

NBS: $\uparrow C 3$ in DBS

$\uparrow C 3$ (pl): $7.7 \mu \mathrm{mol} / \mathrm{L} ; \uparrow M M A$ (ur): $830 \mathrm{mmol} / \mathrm{mol}$ creatn

Propionate uptake (FB) (nmol/10 h/mg prot):

-OHCbl (patient/control): 0.63/1.90

$+\mathrm{OHCbl}$ (patient/control): 1.19/2.34 Mutase (FB): N

Adult with neurological symptoms and low serum vit $\mathrm{B}_{12}$. Improvement after im $\mathrm{OHCbl}$ $\uparrow N$ Hcys (pl): $17 \mu \mathrm{mol} / \mathrm{L}$

Adult with slight anemia and low serum vit B12. Improvement after im $\mathrm{OHCbl}$

$7 y$, anemia since $15 \mathrm{~m}$; low serum vit B12. Improvement after im $\mathrm{OHCbl}$ $\uparrow$ Hcys (pl): $49 \mu \mathrm{mol} / \mathrm{L}$

$2 y$, megaloblastic anemia with low serum vit B12. Improvement after im OHCbl $\uparrow$ Hcys (pl): $39.9 \mu \mathrm{mol} / \mathrm{L}$

$\uparrow M M A$ (ur)

NBS: $\uparrow C 3+C 3 / C 2$ in DBS $\uparrow M M A$ (ur): > $1000 \mathrm{~mol} / \mathrm{mol}$ creatn 
Table 1 Genetic and biochemical findings of validation and discovery cases (Continued)

\begin{tabular}{|c|c|c|c|}
\hline Reference & Gene & $\begin{array}{l}\text { Allele } 1 \text { Nucleotide change } \\
\text { (protein effect) HGMD number }\end{array}$ & $\begin{array}{l}\text { Allele } 2 \text { Nucleotide change } \\
\text { (protein effect) HGMD num }\end{array}$ \\
\hline P7 & $M U T^{a}$ & $\begin{array}{l}\text { c.671_678dupAATTTATG } \\
\text { (p.Val227Asnfs*16) Cl050942 }\end{array}$ & $\begin{array}{l}\text { c.607G > A (p.Gly203Arg) } \\
\text { CM002067 }\end{array}$ \\
\hline P8 & MUT & c.313 T > C (p.Trp105Arg) CM900166 & $\begin{array}{l}\text { c.2150G > T (p.Gly717Val) } \\
\text { CM920488 }\end{array}$ \\
\hline
\end{tabular}

c.2026G > A (p.Ala676Thr)
MUT

c.2026G > A (p.Ala676Thr)

$\begin{array}{lll}\text { P10 } & \text { MUT }^{a} & \text { c.243dupA (p.Pro82Thrfs*2) } \\ \text { P11 } & \text { MUT }^{a} & \begin{array}{l}\text { c.454C }>\text { T (p.Arg152Ter) CM050671 } \\ \text { TCN1 }\end{array} \\ & \text { c.901G > T (p.Asp301Tyr) CM099555 } \\ \text { P12 } & \text { MUT } & \begin{array}{l}\text { c.671_678dupAATTATG } \\ \text { (p.Val227Asnfs*16) Cl050942 }\end{array} \\ \text { P13 } & \text { MUT }^{a} & \text { c.1420C }>\text { T (p. Arg474Ter) CM050685 }\end{array}$

P14

P15

P16

P17
c.671_678dupAATTATG (p.Val227Asnfs*16) Cl050942

c.2026G > A (p.Ala676Thr)
Clinical and biochemical findings

Propionate uptake (FB) (nmol/10 h/mg prot)

-> (mut0)

-OHCbl (patient/control): 0.18/1.16

$+\mathrm{OHCbl}$ (patient/control): 0.11/1.02

Neonatal presentation

$\uparrow M M A$ (ur)

$7 \mathrm{~m}$ metabolic acidosis, lethargy

$\uparrow M M A$ (ur)

NBS: $\uparrow C 3 / C 2$ in DBS

$\uparrow C 3$ (pl): $2.4 \mu \mathrm{mol} / \mathrm{L}$; $\uparrow M M A$ (ur): $772 \mathrm{mmol} / \mathrm{mol}$ creatn

Propionate uptake (FB) (nmol/10 h/mg prot)

- > (mut-)

-OHCbl (patient/control): 0.25/1.16

$+\mathrm{OHCbl}$ (patient/control): 1.16/1.02

c.1084-10A > G (p.?) CS128372 $\uparrow M M A$ (ur)

?

NBS: $\uparrow C 3$ in DBS

Low serum vit B12; $\uparrow N$ MMA (ur): 17-58 mmol/ mol creatn

Neonatal presentation and fatal outcome

$\uparrow M M A$ (ur)

NBS: $\uparrow C 3 / C 2$ in DBS

$\uparrow C 3$ (pl): $3.4 \mu \mathrm{mol} / \mathrm{L}$; $\uparrow M M A$ (ur): $276 \mathrm{mmol} / \mathrm{mol}$

creatn

Propionate uptake (FB) (nmol/10 h/mg prot)

- > (mut-)

-OHCbl (patient/control): 0.28/1.33

+OHCbl (patient/control): 1.13/1.57

c.548A > T (p.His183Leu) CM154654

NBS: $\uparrow C 3 / C 2$ in DBS

$\uparrow C 3$ (pl): $3.64 \mu \mathrm{mol} / \mathrm{L} ; \uparrow M M A$ (ur): $68 \mathrm{mmol} / \mathrm{mol}$ creatn

NBS: $\uparrow C 3+C 3 / C 2$ in DBS

$\uparrow H c y s$ (pl): $18.3 \mu \mathrm{mol} / \mathrm{L} ; \mathrm{N} \mathrm{C3}+$ C4DC (pl); $\uparrow M M A$ (ur): 25-170 $\mathrm{mmol} / \mathrm{mol}$ creatn

NBS: $\uparrow C 3+C 3 / C 2$ in DBS

$\uparrow M M A$ (ur): $2000 \mathrm{mmol} / \mathrm{mol}$ creatn

\section{CM060067}

c.271dupA (p.Arg91Lysfs*14) $\mathrm{Cl} 055013$

c.271dupA (p.Arg91Lysfs*14) $\mathrm{Cl} 055013$

c.748C > T (p.Arg250Ter)

CM081188

c.748C > T (p.Arg250Ter) CM081188

c.1361C > T (p. Ser454Leu) CM032288

c.1678 1681delGAGA (p.Glu560Asnfs*42) CD159487

c.935 T > C (p.lle312Thr) 4d: hypotonia, weight loss, vomiting, $\uparrow$ lac

个Hcys (pl): $214 \mu \mathrm{mol} / \mathrm{L} ; \uparrow C 3: 6.5 \mu \mathrm{mol} / \mathrm{L}+\uparrow C 4 D C$ (pl): $0.25 \mu \mathrm{mol} / \mathrm{L}$ 个MMA (ur): $1197 \mathrm{mmol} / \mathrm{mol}$ creatn

$\uparrow H c y s(p l) ; \uparrow M M A$ (ur)

NBS: $\uparrow C 3+C 3 / C 2$ in DBS

个Hcys (pl): $51 \mu \mathrm{mol} / \mathrm{L} ; \uparrow C 3$ (pl): $4.8 \mu \mathrm{mol} / \mathrm{L} ; \mathrm{MMA}$ (ur): $290 \mathrm{mmol} / \mathrm{mol}$ creatn

NBS: $\uparrow C 3+C 3 / C 2$ in DBS

个Hcys (pl): $59 \mu \mathrm{mol} / \mathrm{L} ; \uparrow C 3$ (pl): $8.5 \mu \mathrm{mol} / \mathrm{L} ; \mathrm{MMA}$ (ur): $1175 \mathrm{mmol} / \mathrm{mol}$ creatn

17 m; anemia, psychomotor delay $\uparrow H c y s$ (pl): $76 \mu \mathrm{mol} / \mathrm{L}$

$10 \mathrm{~m}$ seizures, megaloblastic anemia

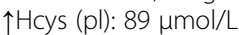

Hypoglycemia at birth, slight PMD during first years

Asymptomatic at $11 \mathrm{y}$

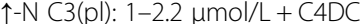

$\uparrow N$ Lac, 3-OHProp, MMA $27 \mathrm{mmol} / \mathrm{mol}$ creatn, MC (ur) 
Table 1 Genetic and biochemical findings of validation and discovery cases (Continued)

\begin{tabular}{|c|c|c|c|c|}
\hline Reference & Gene & $\begin{array}{l}\text { Allele } 1 \text { Nucleotide change } \\
\text { (protein effect) HGMD number }\end{array}$ & $\begin{array}{l}\text { Allele } 2 \text { Nucleotide change } \\
\text { (protein effect) HGMD number }\end{array}$ & Clinical and biochemical findings \\
\hline & & & & $\begin{array}{l}\text { Normal carboxylases activities (FB) } \\
\text { Propionate uptake (FB) (nmol/10 h/mg prot) - > } \\
\text { Normal } \\
\text {-OHCbl (patient/control): } 0.71 / 0.75 \\
+ \text { OHCbl (patient/control): } 0.79 / 1.13\end{array}$ \\
\hline P24 & $\mathrm{ACSF}^{a}$ & c. $424 C>T$ (p.Gln142Ter) & $\begin{array}{l}\text { c.1446_1447delCA } \\
\text { (p.Tyr482Ter) }\end{array}$ & $\begin{array}{l}\text { NBS: } \uparrow M M A \text { (ur) since newborn: } 512 \mathrm{mmol} / \mathrm{mol} \\
\text { creatn } \\
\text { Asymptomatic at } 3 y\end{array}$ \\
\hline P25 & $\mathrm{ACSF}^{a}$ & c.1075G > A (p.Glu359Lys) CM1 16777 & $\begin{array}{l}\text { c.1075G > A (p.Glu359Lys) } \\
\text { CM116777 }\end{array}$ & $\begin{array}{l}\text { 5y PMD, bilateral sensorineural hearing loss } \\
\text { AC (pl): N; } \uparrow M M A \text { (ur): } 360 \mathrm{mmol} / \mathrm{mol} \text { creatn }\end{array}$ \\
\hline P26 & ACSF $^{a}$ & c.1075G > A (p.Glu359Lys) CM116777 & $\begin{array}{l}\text { c.1672C > T (p.Arg558Trp) } \\
\text { CM116840 }\end{array}$ & $\begin{array}{l}\text { Seizures since first months of age } \\
\text { At } 12 \mathrm{y} \text { seizures }+ \text { dystonia } \\
\text { AC (pl): N; } \uparrow M M A \text { (ur): } 124-560 \mathrm{mmol} / \mathrm{mol} \text { creatn } \\
\text { Normal propionate uptake } \pm \mathrm{OHCbl}(\mathrm{FB})\end{array}$ \\
\hline P27 & ACSF3 & c.820C > T (p.Gln274Ter) & c.820C > T (p.Gln274Ter) & $\begin{array}{l}\text { NBS: } \uparrow M M A \text { (ur) since newborn. Asymptomatic at } \\
7 \mathrm{~m}\end{array}$ \\
\hline
\end{tabular}

VC validation case, C3 propionylcarnitine, $C 2$ acetylcarnitine, $C 4 D C$ methylmalonylcarnitine, DBS dried blood spots, $F B$ fibroblasts, $H c y s$ homocysteine, im intramuscular, MMA methylmalonic acid, $\mathrm{OHCb}$ hydroxocobalamin, ur urine, pl plasma, Lac Lactate, $M C$ methylcitrate, NBS newborn screening, $P M D$ psychomotor delay

${ }^{\mathrm{a}}$ Mutations confirmed in parents; ${ }^{\mathrm{b}}$ Variant with uncertain clinical significance; new mutations in bold

Mutations named according to GenBank accession number included in Additional file 3: Table S1

restored by the transfection of patient-derived fibroblasts with a lentiviral construct bearing wild-type SUCLA2 cDNA, the oxygen consumption rate (OCR) of control and patient derived-fibroblasts (transduced or not with the lentiviral construct) was analyzed. In order to force the functioning of the electron transport chain, this experiment was performed in a medium supplemented with galactose. Fig. 2a shows that P23-derived fibroblasts had a diminished bioenergetic profile compared to control cells. Basal respiration was significantly reduced (Fig. 2b). The addition of FCCP to the medium resulted in increased electron flow through the mitochondrial respiratory chain and allowed the maximum respiration rate to be calculated. Figure $2 \mathrm{~b}$ shows this variable to also be significantly reduced in P23 fibroblasts. Oligomycin-sensitive respiration (OSR), which represents the ATP-linked oxygen consumption (Fig. 2c), was also reduced in P23 cells, indicating reduced oxidative phosphorylation.

The transduction of patient dermal fibroblasts with an empty construct ( $\mathrm{Lv}-\mathrm{Co})$ did not influence the bioenergetic profile, except for an increase in the maximum respiration rate which was still below control levels. Transduction with the lentiviral construct bearing

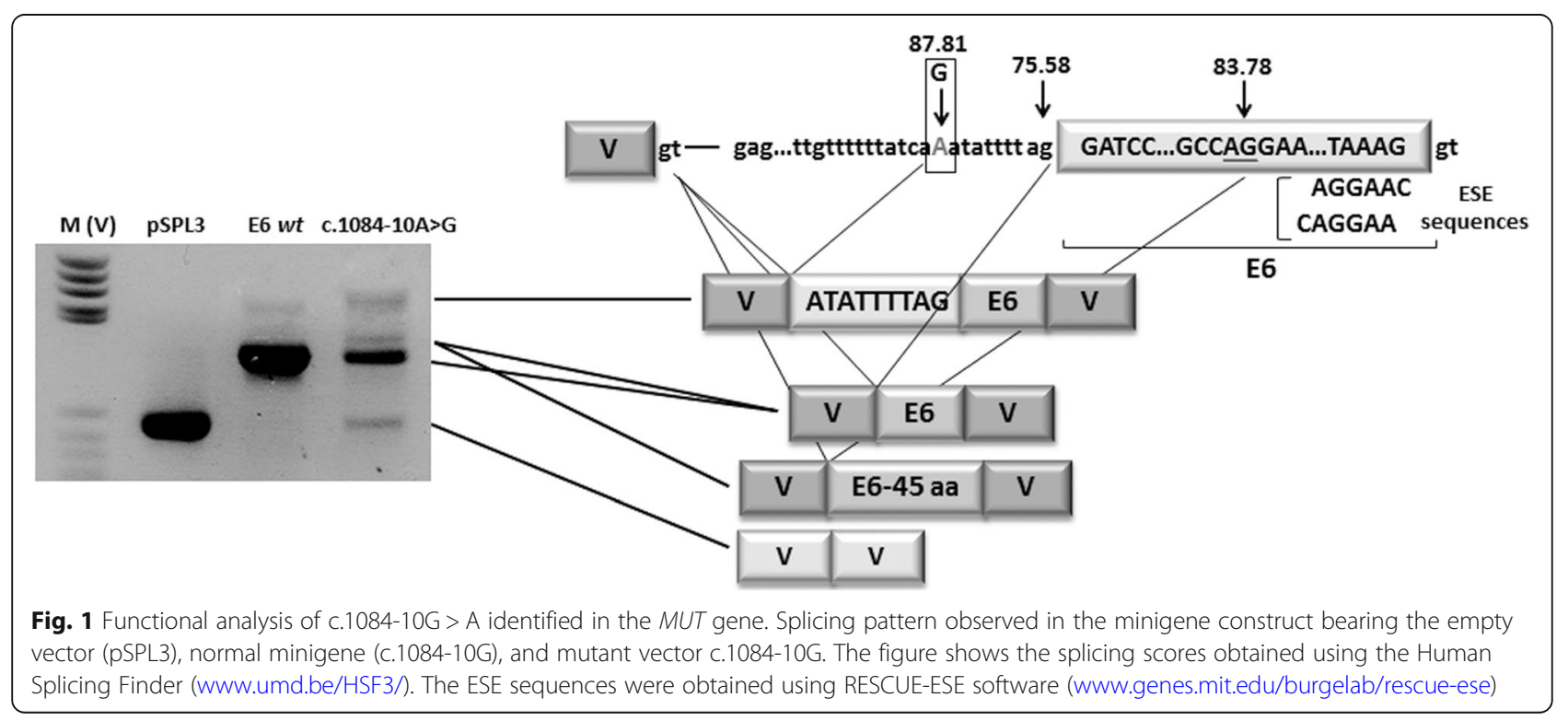



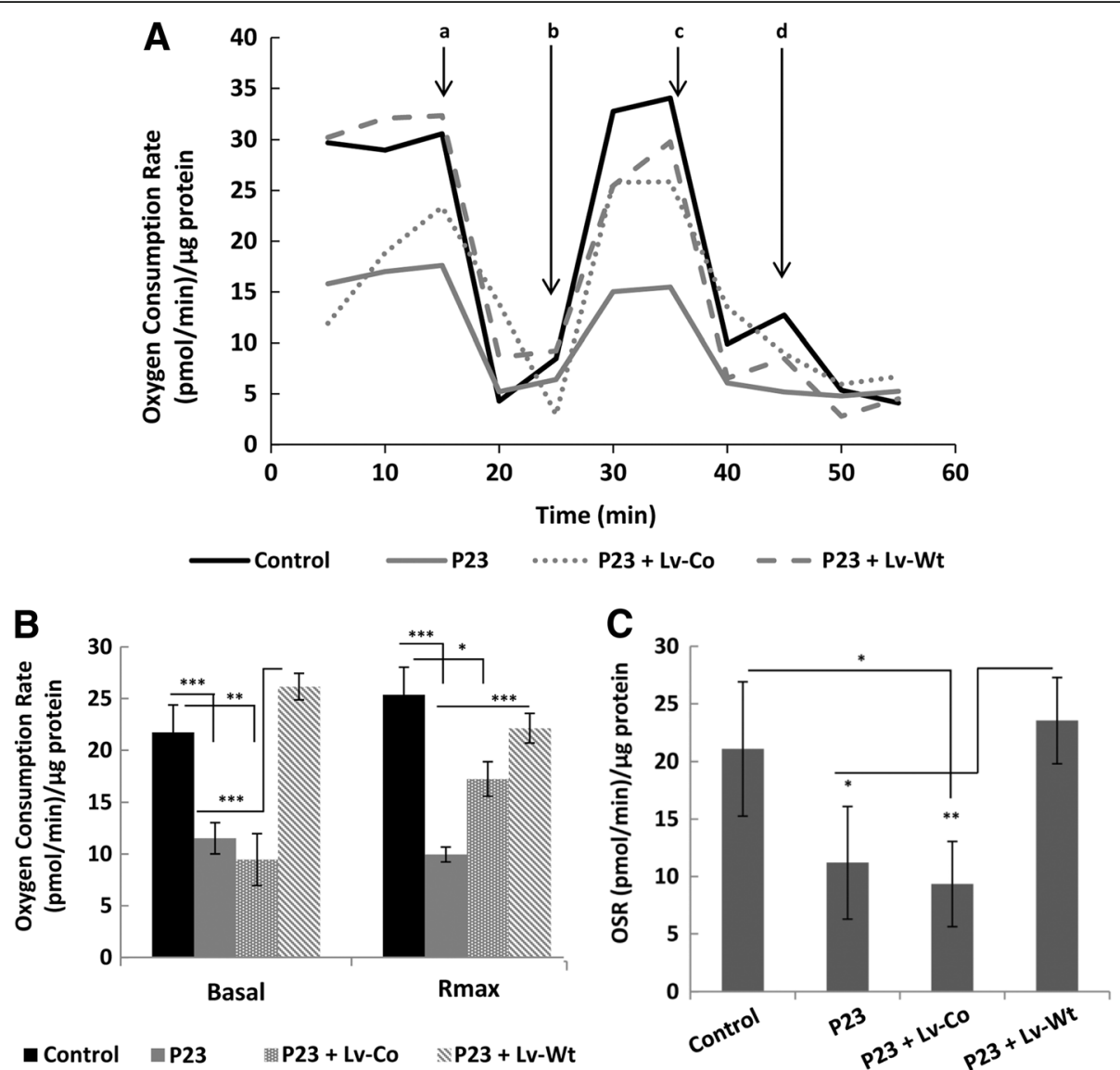

Fig. 2 Functional rescue of mitochondrial dysfunction. Bioenergetic profiles of SUCLA2 in patient P23 fibroblasts transduced with a lentiviral plasmid bearing wild-type SUCLA2 CDNA ( $L v$ Wt) or a null construct ( $L v$ Co), and control-derived fibroblasts. Experiments were performed using a Seahorse XF device. A Oxygen consumption rates of control and patient fibroblasts were measured in DMEM with galactose (1 g/L) instead of glucose, and upon the subsequent addition of a $6 \mu \mathrm{M}$ oligomycin, b $50 \mu \mathrm{M} \mathrm{FCCP,} \mathrm{c} 1 \mu \mathrm{M}$ rotenone or, d $1 \mu \mathrm{M}$ antimycin. B Basal and maximum respiration (Rmax) were calculated for each situation. C Oligomycin-sensitive respiration (OSR) was calculated as the difference between the basal respiration and the oxygen consumption rate measured as described in $(\mathbf{C})$, after the addition of $6 \mu \mathrm{M}$ oligomycin. The results reflect the mean of three biological repetitions. Control values are the mean for two different control cell lines. $\left({ }^{*} p<0.05 ;{ }^{* *} p<0.01 ;{ }^{* * *} p<0.001\right.$ [Student t test])

wild-type SUCLA2 cDNA, however, was able to restore the bioenergetic profile (Fig. 2a). Both basal and maximal respiration rates were restored to control levels (Fig. 2b). As shown in Fig. 2c, oligomycin-sensitive respiration was also significantly increased, suggesting the restoration of oxidative phosphorylation. These results indicate that the bioenergetic deficit observed in the cells from P23 could be caused by defects in SUCLA2.

\section{In silico estimates of the functional impact of missense variants}

Structural analysis of the SUCLA2 variants was performed for prediction of protein dysfunction at the molecular level.

The functional and in silico analyses of the two SUCLA2 variants also suggested them to be damaging. Only AlignGVGD gave a nearly neutral score for
p.Ile312Thr. It should be noted that the pathogenicity score for this variant was closer to the threshold between neutral and damaging replacements, suggesting a milder effect (Additional file 1). This observation agrees with the results of the structural analysis. The wild type residues Gly326 and Ile312 were identified in a homology model of SUCLA2, obtained using pig SUCLG2. Visual analysis of the structures (Fig. 3) showed that both loci fell near the protein's "phosphate shuttle" loop [18], indicating that the replacement of the wild-type residue might interfere with the functional role of this loop. This was particularly noticeable for Gly326, which lies in close contact with residue His299 of the loop, and occurs at a packed location in the structure. The replacement of glycine by the bulkier arginine would undoubtedly create some steric clashes. Gly326 was found to be highly conserved in multiple sequence alignments of the SUCLA2 family, supporting the idea that it may 


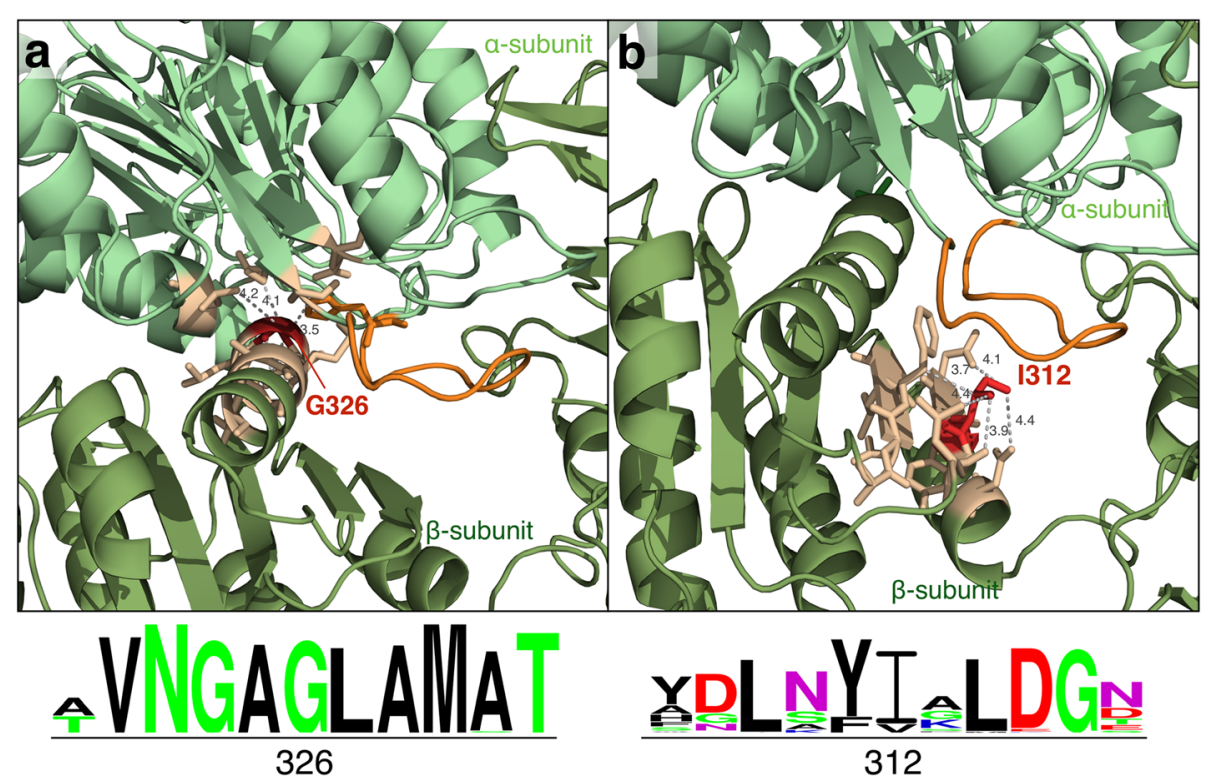

Fig. 3 Structure and conservation analysis of SUCLA2 variants (p.G326R, p.I312T). For each variant, p.G326R (a) or p.I312T (b), the location of the wild-type amino acid (red) and its network of interactions (light orange) are shown. Close residue-residue contacts are drawn with dashed lines. The "phosphate shuttle" loop is marked in dark orange [44]. The conservation pattern for each variant is represented below the structural models. The size of the letters reflects the degree of conservation

have a functional role. Finally, Ile312 was involved in a dense network of residue interactions, although no close contact ( $<5$ Angs) with the residues from the functional "phosphate shuttle" loop was observed. This indicates, once again, that replacement of Ile312 may have a milder impact on function. This agrees with the less strict conservation pattern seen in multiple sequence alignments. In summary, agreement was seen between in silico predictions and structural analyses for both variants, indicating that the observed replacements are pathogenic, although p.Gly326Arg probably causes more severe problems than p.Ile312Thr.

\section{Discussion}

The gold standard for coming to genetic diagnoses of cobalamin defects has for some time been gene-by-gene Sanger sequencing of individual DNA fragments. Enzymatic and cellular methods are employed before such sequencing to help in the selection of the gene defects to be sought, but this is time-consuming and laborious [1]. Further, no biochemical methods have been available to test for cobalamin absorption and transport defects, and while plasma $B_{12}$ concentrations are tentatively used to help in the selection of genes to be sequenced, this has probably led to the under-recognition of cases [34]. This costly, stepwise, and time-consuming methodology is gradually being replaced by NGS technologies, which offer higher throughput and scalability, cost less per sequenced nucleotide and have shorter turnaround times
[35]. The present work reports the NGS analysis of a cohort of patients suspected of having cobalamin defects (plus the corresponding structural/functional analyses for some of the defects involved) and shows it to be quick and reliable.

A $100 \%$ correct diagnostic rate was returned with the validation cohort, with exonic and intronic nucleotide changes and small deletions (c.271dupA in $M M A C H C$ or c.57_64del8 in $M M A D H C$ ) all successfully detected. With the clinical exome massive parallel sequencing system used, the depth and breadth of coverage of the cobalamin metabolism-related genes was >30× and 99\% respectively. The sensitivity and specificity of the method appeared to be very good and the results reproducible (several mutations were detected in more than one unrelated mutant allele i.e., c.271dupA in $M M A C H C$, c.671_678dupAATTTATG in MUT, c.748C $>\mathrm{T}$ in $M M A D H C$ etc.). Thus, even though the use of smaller, specific panels is recommended [36] so that secondary incidental findings are avoided, the present work shows clinical exome sequencing to be successful. It could be used in sequencing analyses of single patients as well groups of patients whose members have other, unrelated disorders.

The LoF mutations detected in GIF and $A M N$ were found to affect the absorption of cobalamin. GIF and $A M N$, in addition to CUBN and TCN1, are not usually captured in the available panels offered by genetic companies but the results reported in the present work have demonstrated they should be analyzed [19, 34]. 
The potential missense changes p.Ala302Pro and p.Ala676Thr, identified in $M U T$, were predicted to be damaging by several bioinformatic algorithms. The present detection of the associated mutations in combination with functional cellular studies provided a rational basis for tailored treatment. Indeed, the cellular uptake of ${ }^{14} \mathrm{C}$-propionate revealed that p.Ala302Pro is a $m u t^{O}$ mutation, while p.Ala676Thr located in the C-terminal of the cobalamin binding domain is likely a $m u t^{-}$mutation. Based on these results, patient P6 might not be responsive to the pharmacological administration of the vitamin, while P9 would likely respond. Case P11 inherited a described LoF mutation in $M U T$ and the functional polymorphism p.Asp301Tyr in TCN1, probably an incidental finding of no clinical significance. This asymptomatic individual was detected in newborn screening. No other mutations were detected in the coding region of MUT, not even in the deep intronic sequence reported to bear an intronic pathogenic mutation [30, 37]. Although massive parallel sequencing exome analysis cannot rule out the presence of other nucleotide changes in promoter or intronic sequences, both the absence of clinical symptoms and the slight abnormal levels of urinary MMA in case P11 suggested he/she was a carrier of the disease.

Variations in genes that do not encode cobalamin metabolism-related proteins (ASCF3 and SUCLA2) were also detected in the present work. Both were associated with slightly increased urinary MMA. The two new variants of SUCLA2 (p.Gly326Arg and p.Ile312Thr) detected are probably damaging changes; both were predicted to be so in bioinformatic and structural analyses. Neither was found to be present in database control populations nor in the in-house control samples. Structural analysis suggested that the replacement of Ile312 may have a mild functional impact, which agrees with the less strict conservation pattern seen in multiple sequence alignments. Importantly, the mitochondrial dysfunction in deficient fibroblasts from P24 was rescued via the stable expression of the SUCLA2 protein through lentiviral transduction. This suggests that SUCLA2 is the gene likely affected in this patient, although his/her symptoms were atypical $[18,38]$. With respect to $A S C F 3$, individuals P24, P25, P26 and P27 were diagnosed as having isolated MMA since the urinary malonic acid that identifies CMAMMA can be easily missed [39]. Given the difficulty in diagnosing CMAMMA, several of the present patients were originally diagnosed during genetic analyses, e.g., exome sequencing [40]. Even though a new method for the rapid metabolic diagnosis of CMAMMA has recently been reported [39], diagnoses by genetic analysis would be improved by second tier genetic testing after newborn screening. In some cases ACSF3 defects only cause biochemical conditions such as short-chain acyl-CoA dehydrogenase deficiency [41] or 3-methylcrotonylglycinuria [42], and symptoms appear only during viral infections etc. [39, 43]. In other cases, as reported in the present work, patients can show neurological damage [19]. Prompt diagnosis would avoid unnecessary treatment with vitamin $B_{12}$.

The use of massive parallel sequencing in a new era of newborn screening would avoid the time-consuming biochemical and enzymatic methods presently used to help identify which genes might be causing isolated MMA, MMA\&HC, HC or CMAMMA. The rapid return of an accurate genetic diagnosis would allow the prescription of an appropriate treatment just a few days after birth. This report illustrates the clinical usefulness of massive parallel sequencing in the diagnosis of cobalamin and related defects. This powerful technology could improve the detection of these under-recognized or rare vitamin $\mathrm{B}_{12}$ defect conditions.

\section{Conclusions}

This work provides evidence supporting the use of massive parallel sequencing as second tier test for confirming cobalamin defects following their detection in newborn screening. Biochemical and cellular analyses and additionally comprehensive phenotyping are needed for an accurate classification of variants for making fully useful clinical reports.

\section{Additional files}

Additional file 1: New and VUS missense mutations in cobalamin genes identified in the discovery cohort. (DOCX $24 \mathrm{~kb}$ )

Additional file 2: Functional prediction of new splicing changes identified in cobalamin gene defects. (DOCX 20 kb)

Additional file 3: Horizontal and vertical coverages of cobalamin and related genes. (DOCX $24 \mathrm{~kb}$ )

\section{Abbreviations}

AdoCbl: Adenosylcobalamin; ATR: Adenosyltransferase; CMAMMA: Combined malonic and methylmalonic aciduria; FCCP: Carbonyl cyanide 4-(trifluoromethoxy) phenyl-hydrazone; Hcys: Homocysteine; MeCbl: Methylcobalamin; MMA: Methylmalonic acid; MMA\&HC: Methylmalonic aciduria with homocystinuria; MMACoA: L-methylmalonyl-CoA; MS: Methionine synthase; MUT: Methylmalonyl-CoA mutase; NGS: Next generation sequencing; OCR: Oxygen consumption rate; TCbIR: Transcobalamin receptor

\section{Acknowledgements}

An institutional grant from the Fundación Ramón Areces to the Centro de Biología Molecular Severo Ochoa, and funding from the European Regional Development Fund, are gratefully acknowledged.

\section{Funding}

This work was funded by grants PI13/01239-PI16/00573; Fundación Isabel Gemio-Fundación La Caixa (LCF/PR/PR16/11110018).

Availability of data and materials

The datasets used and/or analyzed during the current study is available from the corresponding author on request. 


\section{Authors' contributions}

$S B, F L, A V, R N$, MJE carried out the functional and genetic studies; $C R, N P, X C$, participated in the structural studies; MLC, EMH, AM, CP, LPQ, MR, NE, IT IV, RY: clinical diagnosis; follow-up pf the cases; PRP, LR, MU, CPC participated in the biochemical diagnosis; BM and BP conceived the study, participated in its design and coordination, interpreted of results and writing the manuscript. All authors read and approved the final manuscript.

\section{Competing interest}

All authors declare that they have no competing interest.

\section{Ethics approval and consent to participate}

The study was approved by the ethics committee of the Universidad Autónoma de Madrid (CEI48-919). The participants or their legal guardians gave their signed, informed consent to be included.

\section{Consent for publication}

Not applicable

\section{Publisher's Note}

Springer Nature remains neutral with regard to jurisdictional claims in published maps and institutional affiliations.

\section{Author details \\ ${ }^{1}$ Centro de Diagnóstico de Enfermedades Moleculares, Centro de Biología Molecular, Universidad Autónoma de Madrid, CIBERER, IdiPAZ, Madrid, Spain. ${ }^{2}$ Grupo de Bioinformática Translacional Vall d'Hebron Institute of Research (VHIR), Universitat Autònoma de Barcelona, Barcelona, Spain. ${ }^{3}$ ICREA, Barcelona, Spain. ${ }^{4}$ Hospital Clínico Universitario de Santiago, Santiago de Compostela, CIBERER, Santiago de Compostela, Spain. ${ }^{5}$ Hospital 12 de Octubre, Madrid, Spain. ${ }^{6}$ Hospital Universitario La Paz, Madrid, Spain. ${ }^{7}$ Hospital Universitario Niño Jesús, Madrid, Spain. ${ }^{8}$ Hospital Universitario Materno Infantil, CIBEROBN, Universidad de Las Palmas de Gran Canaria, Las Palmas de Gran Canaria, Spain. ${ }^{9}$ Center for Rare Disorders, ASST- Monza, Ospedale San Gerardo, Monza, Italy. ${ }^{10}$ Unidad de Metabolismo Hospital de Niños de La Plata, La Plata, Argentina. ${ }^{11}$ Metabolic Diseases Unit, Lisbon, Portugal. ${ }^{12}$ Hospital Virgen de la Arrixaca, Murcia, Spain. ${ }^{13}$ Hospital Universitario Regional de Málaga, Instituto de Investigación Biomédica de Málaga (IBIMA), Málaga, Spain.}

Received: 18 January 2018 Accepted: 29 June 2018 Published online: 24 July 2018

\section{References}

1. Baumgartner MR, Fowler B. Vitamin B12 Disorders. In: Nenad Blau MD, Michael Gibson K, Dionisi-Vici C, editors. Physicians's guide to the diagnosis, treatment and follow-up of inherited metabolic diseases; 2014. p. 205-17.

2. Banerjee R. B12 trafficking in mammals: a for coenzyme escort service. ACS Chem Biol. 2006;1:149-59.

3. Carmel R, Parker J, Kelman Z. Genomic mutations associated with mild and severe deficiencies of transcobalamin I (haptocorrin) that cause mildly and severely low serum cobalamin levels. Br J Haematol. 2009;147:386-91.

4. Carmel R, Green R, Rosenblatt DS, Watkins D. Update on cobalamin, folate, and homocysteine. Hematology Am Soc Hematol Educ Program. 2003;62-81.

5. Banerjee R, Gherasim C, Padovani D. The tinker, tailor, soldier in intracellular B12 trafficking. Curr Opin Chem Biol. 2009;13:477-84

6. Watkins D, Rosenblatt DS. Update and new concepts in vitamin responsive disorders of folate transport and metabolism. J Inherit Metab Dis. 2012;35:665-70

7. Quadros EV, Lai SC, Nakayama Y, Sequeira JM, Hannibal L, et al. Positive newborn screen for methylmalonic aciduria identifies the first mutation in TCbIR/CD320, the gene for cellular uptake of transcobalamin-bound vitamin B(12). Hum Mutat. 2010;31:924-9.

8. Rutsch F, Gailus S, Suormala T, Fowler B. LMBRD1: the gene for the cblF defect of vitamin B(1)(2) metabolism. J Inherit Metab Dis. 2011; 34:121-6

9. Coelho D, Kim JC, Miousse IR, Fung S, du Moulin M, et al. Mutations in ABCD4 cause a new inborn error of vitamin B12 metabolism. Nat Genet 2012;44:1152-5.
10. Lerner-Ellis JP, Tirone JC, Pawelek PD, Dore C, Atkinson JL, et al. Identification of the gene responsible for methylmalonic aciduria and homocystinuria, cblC type. Nat Genet. 2006;38:93-100.

11. Yu HC, Sloan JL, Scharer G, Brebner A, Quintana AM, et al. An X-linked cobalamin disorder caused by mutations in transcriptional coregulator HCFC1. Am J Hum Genet. 2013;93:506-14.

12. Pupavac M, Watkins D, Petrella F, Fahiminiya S, Janer A, et al. (2016) Inborn error of Cobalamin metabolism associated with the intracellular accumulation of Transcobalamin-bound Cobalamin and mutations in ZNF143, which codes for a transcriptional activator. Hum Mutat. 2016;37(9):976-82.

13. Quintana AM, Yu HC, Brebner A, Pupavac M, Geiger EA, et al. Mutations in THAP11 cause an inborn error of cobalamin metabolism and developmental abnormalities. Hum Mol Genet. 2017;26:2838-49.

14. Gueant JL, Chery C, Oussalah A, Nadaf J, Coelho D, et al. A PRDX1 mutant allele causes a MMACHC secondary epimutation in cblC patients. Nat Commun. 2018;9:67

15. Stucki M, Coelho D, Suormala T, Burda P, Fowler B, et al. Molecular mechanisms leading to three different phenotypes in the cbID defect of intracellular cobalamin metabolism. Hum Mol Genet. 2012;21:1410-8.

16. Dobson CM, Wai T, Leclerc D, Kadir H, Narang M, et al. Identification of the gene responsible for the cblB complementation group of vitamin B12dependent methylmalonic aciduria. Hum Mol Genet. 2002;11:3361-9.

17. Froese DS, Kochan G, Muniz JR, Wu X, Gileadi C, et al. Structures of the human GTPase MMAA and vitamin B12-dependent methylmalonyl-CoA mutase and insight into their complex formation. J Biol Chem. 2010; 285:38204-13.

18. Maas RR, Marina AD, de Brouwer AP, Wevers RA, Rodenburg RJ, et al. SUCLA2 deficiency: a deafness-dystonia syndrome with distinctive metabolic findings (report of a new patient and review of the literature). JIMD Rep. 2016:27:27-32.

19. Pupavac M, Tian X, Chu J, Wang G, Feng Y, et al. Added value of next generation gene panel analysis for patients with elevated methylmalonic acid and no clinical diagnosis following functional studies of vitamin B12 metabolism. Mol Genet Metab. 2016;117:363-8.

20. Sloan JL, Johnston JJ, Manoli I, Chandler RJ, Krause C, et al. Exome sequencing identifies ACSF3 as a cause of combined malonic and methylmalonic aciduria. Nat Genet. 2011;43:883-6.

21. Fowler B, Leonard JV, Baumgartner MR. Causes of and diagnostic approach to methylmalonic acidurias. J Inherit Metab Dis. 2008;31: 350-60.

22. Malvagia S, Haynes CA, Grisotto L, Ombrone D, Funghini S, et al. Heptadecanoylcarnitine (C17) a novel candidate biomarker for newborn screening of propionic and methylmalonic acidemias. Clin Chim Acta. 2015:450:342-8

23. Ng SB, Buckingham KJ, Lee C, Bigham AW, Tabor HK, et al. Exome sequencing identifies the cause of a mendelian disorder. Nat Genet. 2010:42:30-5.

24. Bamshad MJ, Ng SB, Bigham AW, Tabor HK, Emond MJ, et al. Exome sequencing as a tool for Mendelian disease gene discovery. Nat Rev Genet. 2011;12:745-55

25. Yang Y, Muzny DM, Reid JG, Bainbridge MN, Willis A, et al. Clinical whole-exome sequencing for the diagnosis of mendelian disorders. $\mathrm{N}$ Engl J Med. 2013;369:1502-11.

26. Vega Al, Medrano C, Navarrete R, Desviat LR, Merinero B, et al. Molecular diagnosis of glycogen storage disease and disorders with overlapping clinical symptoms by massive parallel sequencing. Genet Med. 2016;18:1037-43.

27. Richards S, Aziz N, Bale S, Bick D, Das S, et al. Standards and guidelines for the interpretation of sequence variants: a joint consensus recommendation of the American College of Medical Genetics and Genomics and the Association for Molecular Pathology. Genet Med. 2015;17:405-24

28. Kleinberger J, Maloney KA, Pollin TI, Jeng LJ. An openly available online tool for implementing the ACMG/AMP standards and guidelines for the interpretation of sequence variants. Genet Med. 2016;18:1165.

29. Forny P, Schnellmann AS, Buerer C, Lutz S, Fowler B, et al. Molecular genetic characterization of 151 Mut-type Methylmalonic Aciduria patients and identification of 41 novel mutations in MUT. Hum Mutat. 2016:37:745-54.

30. Perez B, Rincon A, Jorge-Finnigan A, Richard E, Merinero B, et al. Pseudoexon exclusion by antisense therapy in methylmalonic aciduria (MMAuria). Hum Mutat. 2009;30:1676-82. 
31. Brasil S, Richard E, Jorge-Finnigan A, Leal F, Merinero B, et al. Methylmalonic aciduria cblB type: characterization of two novel mutations and mitochondrial dysfunction studies. Clin Genet. 2015;87:576-81.

32. Fraser ME, Hayakawa $K$, Hume MS, Ryan DG, Brownie ER. Interactions of GTP with the ATP-grasp domain of GTP-specific succinyl-CoA synthetase. J Biol Chem. 2006;281:11058-65.

33. Biasini M, Bienert S, Waterhouse A, Arnold K, Studer G, et al. SWISS-MODEL: modelling protein tertiary and quaternary structure using evolutionary information. Nucleic Acids Res. 2014;42:W252-8.

34. Tanner SM, Sturm AC, Baack EC, Liyanarachchi S, de la Chapelle A. Inherited cobalamin malabsorption. Mutations in three genes reveal functional and ethnic patterns. Orphanet J Rare Dis. 2012;7:56.

35. Xue Y, Ankala A, Wilcox WR, Hegde MR. Solving the molecular diagnostic testing conundrum for Mendelian disorders in the era of next-generation sequencing: single-gene, gene panel, or exome/genome sequencing. Genet Med. 2015;17:444-51.

36. Rehm HL, Bale SJ, Bayrak-Toydemir P, Berg JS, Brown KK, et al. ACMG clinical laboratory standards for next-generation sequencing. Genet Med. 2013;15: 733-47.

37. Rincon A, Aguado C, Desviat LR, Sanchez-Alcudia R, Ugarte M, et al. Propionic and Methylmalonic Acidemia: antisense therapeutics for Intronic variations causing aberrantly spliced messenger RNA. Am J Hum Genet. 2007:81:1262-70

38. Carrozzo R, Verrigni D, Rasmussen M, de Coo R, Amartino H, et al. Succinate-CoA ligase deficiency due to mutations in SUCLA2 and SUCLG1: phenotype and genotype correlations in 71 patients. J Inherit Metab Dis. 2016;39:243-52.

39. de Sain-van der Velden MG, van der Ham M, Jans JJ, Visser G, Prinsen HC, et al. A new approach for fast metabolic diagnostics in CMAMMA. JIMD Rep. 2016;30:15-22.

40. Chu J, Pupavac M, Watkins D, Tian X, Feng Y, et al. Next generation sequencing of patients with Mut methylmalonic aciduria: validation of somatic cell studies and identification of 16 novel mutations. Mol Genet Metab. 2016;118:264-71.

41. Pedersen CB, Kolvraa S, Kolvraa A, Stenbroen V, Kjeldsen M, et al. The ACADS gene variation spectrum in 114 patients with short-chain acyl-CoA dehydrogenase (SCAD) deficiency is dominated by missense variations leading to protein misfolding at the cellular level. Hum Genet. 2008;124:43-56.

42. Lam C, Carter JM, Cederbaum SD, Neidich J, Gallant NM, et al. Analysis of cases of 3-methylcrotonyl CoA carboxylase deficiency (3-MCCD) in the California newborn screening program reported in the state database. Mol Genet Metab. 2013;110:477-83.

43. Alfares A, Nunez LD, Al-Thihli K, Mitchell J, Melancon S, et al. Combined malonic and methylmalonic aciduria: exome sequencing reveals mutations in the ACSF3 gene in patients with a non-classic phenotype. J Med Genet. 2011:48:602-5

44. Matilainen $\mathrm{S}$, Isohanni $\mathrm{P}$, Euro L, Lonnqvist T, Pihko $H$, et al. Mitochondrial encephalomyopathy and retinoblastoma explained by compound heterozygosity of SUCLA2 point mutation and 13q14 deletion. Eur J Hum Genet. 2015;23:325-30.

\section{Ready to submit your research? Choose BMC and benefit from:}

- fast, convenient online submission

- thorough peer review by experienced researchers in your field

- rapid publication on acceptance

- support for research data, including large and complex data types

- gold Open Access which fosters wider collaboration and increased citations

- maximum visibility for your research: over $100 \mathrm{M}$ website views per year

At BMC, research is always in progress.

Learn more biomedcentral.com/submissions 